\title{
Erziehung zur Autonomie als Elternpflicht
}

\author{
Von MONIKA BETZLER (Bern)
}

Einer weit geteilten, der herkömmlichen Moral verpflichteten Auffassung zufolge haben Eltern besondere Pflichten gegenüber ihren eigenen Kindern. Das heißt, sie haben Pflichten qua ihrer Elternschaft, die andere Personen, die keine Eltern sind, nicht haben. Zu diesen Pflichten gehören unter anderem Fürsorge, Liebe sowie die Erziehung ihrer Kinder zur Autonomie. Diesen Pflichten entsprechen bestimmte Rechte der Kinder. So haben Kinder unter anderem sowohl ein Recht darauf, dass ihre physischen und emotionalen Bedürfnisse befriedigt werden, als auch ein Recht auf ein eigenes Leben. Wer als Eltern die Rechte seiner Kinder missachtet, handelt moralisch falsch. Dies zeigt sich unter anderem daran, dass wir Eltern tadeln, die sich nicht (oder nicht hinreichend) um das Wohlergehen ihrer Kinder kümmern, sie ohne emotionale Zuneigung behandeln oder sie in ungebührender Weise fremdbestimmen. Um den Gehalt dieser elterlichen Pflichten besser zu verstehen, bedarf es eines genaueren Verständnisses von Fürsorge, Liebe und Autonomie.

Im Folgenden werde ich mich jedoch ausschließlich mit der Elternpflicht befassen, die darin besteht, sein Kind zur Autonomie zu erziehen. ${ }^{1}$ Damit möchte ich nicht nahe legen, dass diese Pflicht ausschließlich den Eltern zukommt. So obliegt es unter anderem auch schulischen Institutionen, die Autonomie von Kindern zu befördern. Die Pflicht, sein Kind zur Autonomie zu erziehen, kommt Eltern jedoch aus zwei Gründen in besonderem Maße zu. Eltern sind auf Grund der Nahbeziehung zu ihren Kindern, die sie normalerweise haben, zum einen am besten in der Lage, das Recht des Kindes auf ein eigenes Leben zu berücksichtigen. Sie sind zum andern auf Grund der Tatsache, dass es jeweils $i h r$ Kind ist und sie mit ihrer Elternschaft eine besondere Verantwortung für ihr Kind übernommen haben, in ebenso distinkter Weise in der Pflicht, die Autonomie des Kindes zu befördern. Dies heißt jedoch nicht, dass die Pflicht, das eigene Kind zur Autonomie zu erziehen, stärker ist als andere Elternpflichten. Es handelt sich lediglich um eine Pflicht, die Eltern unter anderen haben. Das Verhältnis der verschiedenen Elternpflichten zueinander kann ich im Rahmen dieses Beitrags nicht klären. Es geht mir im Folgenden nur darum zu erläutern, worin der Gehalt der Elternpflicht zur AutonomieErziehung genauer besteht. Weshalb gibt es diese Pflicht, und was legt sie nahe?

Auch wenn noch völlig offen bleibt, was genau Autonomie bedeutet, die zu befördern die Pflicht von Eltern ist, so scheint Folgendes intuitiv einleuchtend: Kindern muss in ihrer Entwicklung dazu, ein eigenes Leben zu führen, geholfen werden. Kinder sind nicht nur unvollkommen im rationalen Überlegen und in der angemessenen Wahrnehmung von Gründen.

1 Vgl. zum Beispiel Brighthouse (1998), 719-745, der für diese Elternpflicht argumentiert. 
Sie kennen darüber hinaus viele Tatsachen und Sachverhalte nicht, verstehen noch nicht hinreichend die Implikationen ihres Tuns, können längerfristige Konsequenzen ihres Handelns nicht abschätzen und sind wenig realistisch in der Einschätzung ihrer Kompetenzen. Sowohl die Unkenntnis über sich und die Welt als auch die noch nicht ausgebildete Fähigkeit zum Überlegen erklären, warum sie (noch) in defizitärer Weise ihre Entscheidungen treffen und sich in dem Prozess des Entscheidens häufig irren. ${ }^{2}$ Damit hängt zusammen, dass Kinder noch nicht über ein hinreichend diachrones Verständnis ihrer selbst und ihres Tuns verfügen.

Aber abgesehen von ihrer epistemischen und deliberativen Schwäche verfügen sie auch noch nicht über hinreichend gefestigte volitionale beziehungsweise evaluative Einstellungen. Sie wissen noch nicht, was sie ,wirklich“ wertschätzen, können nicht recht bemessen, was wertvoll ist, verfügen noch kaum über stabile Präferenzen und sind nicht oder noch unzureichend in der Lage, ihre Neigungen und Motive kritisch zu hinterfragen. Vielmehr schwanken Kinder stark auf Grund unmittelbarer Eindrücke und sozialen Drucks von meist Gleichaltrigen in dem, was ihnen wichtig ist. Darüber hinaus verstehen sie sich nicht als Personen mit einer bestimmten evaluativen Perspektive.

Was genau sollen also Eltern vor diesem Hintergrund fördern? Was kann mit Autonomie überhaupt gemeint sein? Einem weit verstandenen Begriff von Autonomie zufolge ist eine Person nur dann autonom, wenn sie zwei Bedingungen erfüllt, die diesen soeben skizzierten epistemischkognitiven und volitional-evaluativen Fähigkeiten entsprechen. ${ }^{3}$ Sie muss zum einen ein „Selbst““ ausgebildet haben. Demzufolge muss sie motivationale und evaluative Einstellungen haben, die in einem emphatischen Sinne ihre eigenen sind. Nur dann kann sich eine Person als jemand verstehen, dem bestimmte Dinge wichtig sind, die daher ein bestimmtes Verständnis ihrer selbst erwirbt und damit zusammenhängend eine bestimmte evaluative Perspektive ausbildet, die sie als Person normativ charakterisiert. Die Intuition, die dieser Bedingung zu Grunde liegt, ist die Folgende: Nur was einer Person selbst wirklich eigen ist - viele sprechen in diesem Zusammenhang davon, dass sie sich mit einer ihrer Einstellungen, ,identifiziert“" oder dass eine ihrer Einstellungen ein besonderer Ausdruck dessen ist, was ihr eigen ist -, kann eine begründende Auskunft darüber geben, wie sie sich zu verstehen hat und wer sie sein möchte. Andernfalls würde sie ein Leben führen, das nicht ihres ist. Sie würde sich entfremdet und damit als passiver Zuschauer dessen fühlen, was sie tut. Ich bezeichne dies als die Authentizitätsbedingung der Autonomie. ${ }^{4}$

Zum andern können die Einstellungen, die der Person eigen sind, nur dann zu ihrem Selbstverständnis und ihrer evaluativen Perspektive beitragen, wenn sie sich im Lichte dieser Einstellungen über die Zeit hinweg zu orientieren vermag. Diese Einstellungen müssen daher nicht nur ihr eigen sein, sondern sich sowohl als stabil als auch im Lichte etwaiger Veränderungen und anderer Einstellungen als hinreichend flexibel erweisen. Neben ihren evaluativen Fähigkeiten benötigt sie daher deliberative und rationale Fähigkeiten, um Kohärenz in ihren Einstellungen zu erzielen, über angemessene Mittel zur Realisierung ihrer für wertvoll gehaltenen Zwecke nachzudenken und die Implikationen ihrer wertgeschätzten Zwecke abzuschät-

2 Vgl. Brennan (2002), 53-69, die betont, dass Kinder schlechte Entscheidungen treffen und sich in den Entscheidungsprozessen häufig täuschen.

3 Diese beiden Bedingungen habe ich ausgeführt in: Betzler (2009/2011), $177 \mathrm{f}$.

4 In einer früheren Arbeit spreche ich von der Wertungsbedingung von Autonomie (Betzler 2009/2011, 177). In Anlehnung an die in der Literatur üblichere Formulierung dieser Bedingung verwende ich hier den Begriff der Authentizität (vgl. zuletzt Dworkin 2010, Kap. 11). Für Autonomie relevanter als die im Begriff der „Authentizität“ mitschwingende Intuition, dass eine Person sich selbst treu zu sein hat, scheint mir die Intuition, dass eine Person in der Lage sein muss, Dinge von Wert tatsächlich wertzuschätzen und sich im Lichte ihrer Wertschätzungen zu verstehen (vgl. Christman 2009, Kap. 7). 
zen. Sie benötigt epistemische und emotionale Fähigkeiten, um neue Gründe zu erkennen und ihre ihr eigenen motivationalen und evaluativen Einstellungen im Lichte neuer Gründe zu überdenken. Dazu muss sie in der Lage sein, momentane, sie überfallende Neigungen von ihren eigenen Einstellungen zu unterscheiden und erstere gegebenenfalls zu kontrollieren. Andernfalls würde sie ein instabiles und vor allem nicht kohärentes Leben führen. Ich bezeichne dies als die Kontrollbedingung von Autonomie. ${ }^{5}$

Die Authentizitäts- und Kontrollbedingung sind für den Begriff der Autonomie zentral, wenn auch unterschiedliche Theorien der Autonomie diese unterschiedlich spezifizieren. Zum einen wird ganz unterschiedlich konzeptualisiert, was es heißt, „eigene“ Einstellungen zu haben, sowie was es heißt, sich zu kontrollieren oder sich im Lichte seiner eigenen Einstellungen zu führen. Aber abgesehen davon, dass die beiden Bedingungen unterschiedlich gedeutet werden, vertreten manche Theoretiker und Theoretikerinnen der Autonomie darüber hinaus die Auffassung, dass eine Person über ein hinreichendes Selbstwertgefühl verfügen, hinreichend viele Optionen besitzen und in einem sozialen Gefüge mit persönlichen Nahbeziehungen eingebunden sein muss, um diese beiden Bedingungen überhaupt erfüllen zu können. Im Folgenden lege ich mich nicht auf eine bestimmte Theorie der Autonomie fest, sondern gehe von einem weiten, von rivalisierenden Theorien geteilten, Kernbegriff der Autonomie aus. Demzufolge muss eine Person Einstellungen haben, die zum Ausdruck bringen, dass ihr etwas eigen ist. Häufig wird in diesem Zusammenhang auch davon gesprochen, dass ihr etwas ,wirklich“ wichtig ist oder sie etwas „wirklich“ will. Und sie muss Fähigkeiten haben, sich auch über die Zeit hinweg an diesen Einstellungen zu orientieren. Kurz: Zum Kernbegriff der Autonomie gehört, dass eine Person die Authentizitäts- und Kontrollbedingung erfüllen muss.

Wenn wir uns vor diesem Hintergrund die Frage stellen, inwiefern Kinder autonom werden können, so möchte ich weder ein statisches Verständnis von Kindheit nahe legen noch suggerieren, dass Kindheit in erster Linie als ein defizitäres Stadium auf dem Weg zum Erwachsenenalter zu betrachten ist. Tatsächlich lässt sich der Begriff des Kindes auf Menschen mit ganz unterschiedlich ausgebildeten Fähigkeiten anwenden. So verfügt ein Kleinkind weder über rationale noch über evaluativ-volitionale Fähigkeiten, sondern wird allein durch seine unmittelbaren Neigungen motiviert. Eine 15-Jährige hingegen ist sowohl zu rationaler Überlegung als auch zu Wertungen - zumindest in gewissem Maße - in der Lage. Entsprechend scheint sich auch die Autonomie eines Kindes vom Kleinkindstadium zur Adoleszenz zu entwickeln. Autonomie ist hierbei eine Eigenschaft, die Kindern graduell zugeschrieben werden kann. Wenn es also darum geht zu präzisieren, worin genau die elterliche Pflicht besteht, die Autonomie des eigenen Kindes zu befördern, muss der Entwicklungsstand des jeweiligen Kindes berücksichtigt werden. Dass Kinder hinsichtlich ihrer Autonomie defizitär sind, schließt hierbei nicht aus, dass Kindheit gegenüber dem Erwachsensein in anderen Hinsichten ein genuiner Wert zukommen kann.

Die Elternpflicht zur Beförderung der Autonomie eines Kindes kann hierbei sowohl negativer als auch positiver Art sein. So kann ein Kind als Erwachsener kein eigenes Leben führen, wenn Eltern es in unangemessener Weise fremdbestimmen. Es kann aber auch kein eigenes Leben führen, wenn Eltern ihm nicht dabei helfen, autonom zu werden. Eltern dürfen sich daher nicht in jeder erdenklichen Weise einmischen. Sie müssen aber auch in bestimmter Weise handeln, um die Autonomie ihres Kindes zu befördern. Wir müssen daher herausfinden, inwiefern Eltern die Eigenschaften und Fähigkeiten des Kindes respektieren müssen und inwiefern sie die genannten Schwächen des Kindes helfen können zu überwinden. Hierbei geht es jedoch nicht darum, ein Kind zu maximaler Autonomie zu erziehen. Das Erziehungs-

$5 \mathrm{Zu}$ den dem Autonomie-Begriff in seinem weiten Sinn unterliegenden Intuitionen vgl. Oshana (1998), 81 f.; dies. (2003), 99 ff. 
ziel ist nicht die einem perfektionistischen Ideal verpflichtete Autonomie, sondern die Fähigkeit des Kindes, selbst eigene Einstellungen zu erwerben und sich entsprechend zu orientieren. Wir müssen also herausfinden, inwiefern Eltern dazu beitragen können, dass ein Kind die Authentizitäts- und Kontrollbedingung von Autonomie zu erfüllen vermag.

\section{Das Rätsel: Autonomie durch Fremdbestimmung}

Wenn wir davon ausgehen, dass Eltern dem Entwicklungsstand ihres Kindes entsprechend die Pflicht haben, die Autonomie ihres Kindes zu befördern, so führt diese Forderung zu einem Problem, das ich als das ,Rätsel der Erziehung zur Autonomie“ bezeichnen möchte. ${ }^{6}$ Es ergibt sich aus den folgenden Annahmen:

(i) Kinder sind nicht autonom und müssen lernen, autonom zu werden.

(ii) Respekt vor der Autonomie einer anderen Person erfordert, dass man bestimmte Handlungen und Verhaltensweisen gegenüber dieser Person unterlässt und sie in ihren Wertungen und in ihrer Selbstkontrolle nicht beeinflusst.

(iii) Eltern haben die Pflicht, die Autonomie ihrer Kinder zu befördern.

(iv) Da Kinder nicht autonom sind, dürfen andere Personen mit ihnen anders umgehen, sie bevormunden, ihnen sagen, was richtig und wertvoll ist, und sie auf diese Weise fremdbestimmen.

Aus diesen Annahmen ergibt sich das Problem, wie Eltern die Autonomie ihres Kindes befördern und damit respektieren können, wenn sie zugleich auf Grund der mangelnden Autonomie ihres Kindes dazu angehalten sind, sich ständig in die Angelegenheiten des Kindes einzumischen und es auf diese Weise fremdzubestimmen. Kurz: Wie kann Autonomie mittels Fremdbestimmung möglich werden? Ist der Versuch, die Autonomie eines Kindes durch die Einmischung der Eltern und somit durch einen nicht-autonomen Prozess zu befördern, nicht von vornherein zum Scheitern verurteilt? ${ }^{7}$

Tatsächlich bevormunden Eltern vor allem kleinere Kinder in vielfältiger Weise. Sie bestimmen zum Beispiel, was zu tun ist, ohne das Kind um Einwilligung zu fragen. Sie tun Dinge gegen den expliziten Willen des Kindes. Und sie geben ihm vor, was wertvoll ist, versuchen das Kind zur Übernahme bestimmter Einstellungen zu bewegen und übernehmen Entscheidungen sowie die Planung seines Lebens. Sie beschränken seine Optionen, teilen nicht jede Information mit ihnen, sanktionieren sein Verhalten und zeigen ihm Grenzen auf.

Bei näherer Betrachtung ist jedoch nicht jede Form der Einmischung autonomie-unterminierend. Um dies zu zeigen, bedarf es einer Unterscheidung zwischen Autonomie als Zustand und Autonomie als Fähigkeit. Diese Unterscheidung wird deutlich, wenn wir die attributive, prädikative und adverbiale Verwendung der Eigenschaft ,,autonom“ näher betrachten. Wenn Autonomie attributiv verstanden wird - und etwa vom ,autonomen Kind“ die Rede ist -, dann

6 Cuypers u. Haji (2007), 82, sprechen in diesem Zusammenhang vom einem Problem der Authentizität, das sich für die Erziehung zur Autonomie stellt.

7 Vgl. Noggle (2005), 101, der von einem Paradox der Selbst-Erschaffung spricht. Kinder können sich nicht einfach aus dem Nichts selbst kreieren. Noggle hält dies jedoch für wenig problematisch, da die Einstellungen, die sie langsam erwerben, zwar häufig von außen vorgegeben werden und insofern fremdbestimmt sind. Aber da Kinder noch kein wahres Selbst haben, können diese dann erworbenen Einstellungen nicht als inauthentisch abgelehnt werden. 
wird dem Kind ein Zustand attestiert, in dem Kinder per definitionem nicht zu sein scheinen. Wenn einem Kind die Eigenschaft der Autonomie zukommt und es somit in einem autonomen Zustand ist, scheint es kein Kind mehr zu sein. Bei der attributiven Verwendung handelt es sich folglich um eine contradictio in adjecto.

Wird die Zuschreibung von Autonomie jedoch prädikativ verstanden, so können wir etwa sagen, dass ein Kind autonom ist, aber auch dass ein Kind autonom wird. Ein Kind kann hierbei autonom werden, wenn es seine eigenen Fähigkeiten zur Autonomie aktualisiert. Dies wird besonders in der adverbialen Verwendung deutlich, wenn wir davon sprechen, dass ein Kind schon richtig autonom spielt oder andere Dinge autonom tut. Damit kann unter anderem gemeint sein, dass es Fähigkeiten aktualisiert, die es graduell in einen autonomen Zustand versetzen. Die Frage ist vor diesem Hintergrund, welcher Art diese Fähigkeiten sind, die dem Kind dazu verhelfen, autonom zu werden und letztendlich ein autonomer Erwachsener zu sein. Eine Antwort auf diese Frage würde zeigen, inwiefern die Einmischung von Seiten der Eltern nicht autonomieunterminierend sein muss.

Eine solche Einmischung würde zum Beispiel den Erwerb der Autonomie eines Kindes untergraben, wenn Eltern entweder die für Autonomie kausal ermöglichenden Bedingungen nicht schaffen, oder wenn sie ihrem Kind keinen Raum lassen, die Authentizitäts- und Kontrollbedingung von Autonomie selbst erfüllen zu lernen. So können sie - was die ermöglichenden Bedingungen anlangt - den Erwerb der Autonomie beeinträchtigen, wenn sie zum Beispiel das Selbstwertgefühl des Kindes durch verbale Demütigungen oder unangemessene Strafen untergraben. Das Gleiche gilt für die Möglichkeit, einem heranwachsenden Kind Handlungsoptionen bereitzustellen und ihm dadurch eine „offene Zukunft ${ }^{\text {"6 }}$ in Aussicht zu stellen, sowie für den sozialen Rahmen von gelebten Nahbeziehungen zu sorgen, in denen Kinder Vertrauen und Geborgenheit erfahren. Was die Möglichkeit anlangt, dem Kind Raum zur Erfüllung der Authentizitäts- und Kontrollbedingung zu eröffnen, so würde dies durch folgendes bevormundendes Verhalten beeinträchtigt oder gar verunmöglicht: Die Authentizitätsbedingung kann von einem Kind nur schwerlich im Laufe seiner Entwicklung erfüllt werden, wenn Eltern die eigenen Wertvorstellungen nicht nur vorleben, sondern entweder durch Missachtung der eigenen Neigungen des Kindes oder durch anderweitige Sanktionsmechanismen - wie Ächtung alternativer Wertvorstellungen - das Kind darin behindern, eigene wertende Einstellungen auszubilden. Darüber hinaus können Eltern es unterlassen, die deliberativen Fähigkeiten eines Kindes zu fördern, und es darin behindern, Gründe als für das Kind relevant zu erkennen. Dies ist dann der Fall, wenn Eltern auch dann noch alles entscheiden, wenn das älter gewordene Kind bereits mehr kognitive, epistemische und evaluative Fähigkeiten ausgebildet hat und in seinem Rahmen selbst Entscheidungen treffen kann.

Elterliche Fremdbestimmung ist nur dann im strengen Sinne bevormundend und somit autonomieuntergrabend, wenn es von Eltern unterlassen wird, die Fähigkeit des Kindes zur Selbstbestimmung, die sich mit zunehmendem Alter und mit zunehmender Entwicklung ausbildet, zu berücksichtigen. Diese Fähigkeit zu berücksichtigen impliziert, dass ein Kind durch seine Eltern ermuntert wird, eigene Einstellungen auszubilden und seine rationalen Fähigkeiten zu üben. Die Autonomie seines Kindes zu befördern, kann daher nicht heißen, seine Fähigkeiten zur Autonomie zu untergraben.

Dem oben formulierten Rätsel unterliegt eine statische Auffassung von Autonomie, der zufolge eine Person entweder im Zustand der Autonomie ist oder nicht. Wenn man jedoch berücksichtigt, dass Kinder relevante Fähigkeiten besitzen, um autonom zu werden, dann muss sich die elterliche Pflicht darauf richten, diese Fähigkeiten zu befördern. Sie kann nicht

$8 \quad$ Vgl. Feinberg (1980), 148-151. 
darin bestehen, ein Kind auf Grund der in seinem momentanen Zustand abgesprochenen Autonomie in jeder Hinsicht fremdzubestimmen.

Man könnte allerdings einwenden, dass Eltern ein Kind fremdbestimmen, selbst wenn sie sich bemühen, seine Fähigkeiten zur Autonomie zu befördern. Dies, so der Einwand, tun sie dadurch, dass sie (ebenso wie das gesamte soziale Umfeld) dem Kind Wertungen vorgeben. Das Kind könne daher gar nicht zu ihm eigenen Wertungen gelangen und somit die Authentizitätsbedingung nie erfüllen. Dieser Einwand und die damit einhergehende Verschärfung des Rätsels scheint mir jedoch voreilig. Im Folgenden möchte ich zeigen, auf welche Weise Eltern die Fähigkeit ihres Kindes zur Autonomie befördern können, und zwar indem sie ihnen die Möglichkeit geben, eigene Wertungen auszubilden. Denn erst wenn ein Kind eigene Wertungen auszubilden vermag, kann es auch zunehmend lernen, sich im Lichte dieser ihm eigenen Wertungen zu orientieren.

\section{Auf dem Weg zur Authentizität}

Wie können Eltern ihrem Kind helfen, wertende Einstellungen zu erwerben, die in einem emphatischen und autonomiebefördernden Sinn seine eigenen sind? Schließlich weiß ein Kind nicht, was gut für es ist, beziehungsweise nur in unzulänglicher, irrtumsanfälliger Weise. Es kann sich ebenso nur unzulänglich auf die eigene Zukunft und auf seine gegebenen Einstellungen beziehen, und es hat noch keine evaluative Perspektive erworben. Ebenso ist es noch nicht oder nur unzureichend in der Lage, ihm eigene Wertungen von momentanen Wünschen, Gefühlen und anderen Motiven zu unterscheiden. Ich habe bereits erwähnt, dass ich mich im Rahmen dieses Beitrags nicht mit den verschiedenen Vorschlägen zur Deutung der Authentizitätsbedingung kritisch auseinandersetzen kann. Ich werde mich vielmehr darauf beschränken, eine eigene Lesart dieser Bedingung vorzuschlagen. Meiner Lesart zufolge erwirbt ein Kind Einstellungen, die ,ihm eigen“ genannt werden können, wenn es lernt, selbst wertzuschätzen. ${ }^{9}$ Um dies zu verdeutlichen, möchte ich auf die von Samuel Scheffler vorgestellte Analyse der Einstellung des Wertschätzens Bezug nehmen.

Etwas wertzuschätzen ist Scheffler zufolge ein Komplex von Einstellungen. Ein X wertzuschätzen, impliziert:

(i) die Überzeugung, dass X wertvoll oder gut ist;

(ii) eine Neigung, eine Reihe von kontextabhängigen Emotionen bezüglich X zu empfinden;

(iii) eine Disposition, diese Emotionen als verdient oder angemessen zu erfahren;

(iv) eine Disposition, bestimmte X-bezogene Überlegungen als Handlungsgründe in relevanten deliberativen Kontexten zu betrachten. ${ }^{10}$

Die erste Bedingung, der zufolge eine Person eine Überzeugung darüber hat, dass das, was wertgeschätzt wird, auch wertvoll ist, setzt voraus, dass man über hinreichend Wissen über das, was gut beziehungsweise wertvoll ist, verfügt. Die zweite Bedingung bringt zum einen die Bindung eines Akteurs an das, was für wertvoll befunden wird, zum Ausdruck. Im Gegensatz zu einer bloßen Überzeugung, dass etwas wertvoll ist, ist die Person, die etwas wertschätzt, affizierbar gegenüber dem, was sie wertschätzt. So mag sie zum Beispiel geneigt sein,

9 Ich verwende die Verben „,wertschätzen“ und „,werten“ synonym.

10 Vgl. Scheffler (2010), 29. 
Angst zu empfinden, wenn das, was sie wertschätzt, auf Grund äußerer Umstände bedroht ist. Sie mag Freude empfinden, wenn das von ihr Geschätzte allseitige Zustimmung findet. Dass eine Person jedoch in diesem Sinne verwundbar und damit abhängig ist von dem, was sie wertschätzt, hängt damit zusammen, dass sie ein Verständnis ihrer selbst mit einer normativen Identität und damit mit einer bestimmten evaluativen Perspektive entwickelt hat. Sofern sie sich dieser Perspektive bewusst ist und weiß, dass das, was sie so wertet, wertvoll ist, erfährt sie ihre Emotionen als angemessen. Sie erfährt damit auch ihre Verwundbarkeit als angemessen. Dies drückt die dritte Bedingung aus. Wer sich etwa als jemand versteht, der Musik wertschätzt, der hat nicht nur in der Vergangenheit zahlreiche musikalische Erfahrungen gemacht (um nur ein Beispiel herauszugreifen). Die Person sieht sich auch als eine solche, der diese Erfahrungen wichtig sind. Dies erklärt schließlich die vierte Bedingung, der zufolge sie Überlegungen, die sich auf ihre Wertschätzung von Musik beziehen, in relevanten deliberativen Kontexten berücksichtigt. Das heißt, sie betrachtet vor dem Hintergrund ihrer normativen Identität diese Tatsache als Grund, öfters ins Konzert zu gehen oder auf eine teure Sportart zu verzichten, um Zeit und finanzielle Mittel für das Klavierspiel zu haben. Dies tut sie unter anderem auch, weil sie eine Zukunft hat, die sie als die ihre betrachtet.

Wenn diese Beobachtungen ebenso wie Schefflers Analyse richtig sind, hat eine Person, die wertschätzt, eine eigene evaluative Perspektive, sie macht sich bestimmte wertvolle Eigenschaften dadurch zu Eigen, dass sie sich als emotional empfänglich für sie zeigt und sich entsprechend zu führen vermag. Darüber hinaus ist sie nicht einfach nur blind empfänglich, sondern kann ihre Empfänglichkeit als Ausdruck ihrer Konzeption dessen, was gut ist, verstehen und sich insofern als jemand betrachten, der sich durch eigene Gründe leitet. Ich gehe hierbei davon aus, dass diese Deutung der Authentizitätsbedingung Vorteile gegenüber alternativen Konzeptionen hat und wesentliche Intuitionen einfängt, die wir damit verbinden.

Es scheint jedoch so, dass zumindest kleinere Kinder nicht einmal die Voraussetzungen erfüllen, in dem oben definierten Sinn die Einstellung des Wertens einzunehmen. Sie scheinen also keine eigenen Einstellungen haben zu können. Was wäre daher die Pflicht der Eltern, um zu befördern, dass Kinder selbst etwas wertschätzen und in diesem Sinne ihnen eigene Einstellungen erwerben? Die erste Frage betrifft vor allem die Voraussetzungen, die Kinder selbst mitbringen, um Wertschätzen zu lernen. Die zweite Frage betrifft den spezifischen Gehalt der Elternpflicht. Zunächst möchte ich mich den Voraussetzungen zuwenden, die Kinder selbst mitbringen, um eine eigene Einstellung des Wertschätzens zu erwerben. Wie sich zeigen wird, ist es nämlich nicht so, dass Kinder überhaupt keine Fähigkeit besitzen, eine stabilere evaluative Perspektive zu entwickeln.

Kinder können lernen, etwas wertzuschätzen, da sie bereits über eine Proto-Form des Wertschätzens verfügen. Es handelt sich um eine Proto-Form, da sie weder evaluatives Wissen noch Selbstbezüglichkeit auf die eigene Zukunft und ein Verständnis der eigenen Identität voraussetzt. Diese Proto-Form, die weder reflexiv ist noch Rechtfertigungsbedingungen unterliegt, teilt jedoch die folgenden Eigenschaften mit der soeben charakterisierten Einstellung des Wertschätzens: Sie ist diachron und stabil und nicht, wie im Fall bloßer Wünsche, momentan und volatil. Dies liegt unter anderem daran, dass sie aus wiederkehrenden, auf konstante Dinge oder Sachverhalte gerichteten emotionalen Verbindungen besteht und auf Grund dieser diachronen Stabilität bereits in einer Form zum Ausdruck bringt, was der Person, die diese Einstellung hat, wichtig ist. Diese Einstellung, die ich als Proto-Form des Wertschätzens individuieren möchte, wird „Caring“ oder „sich Kümmern“" genannt. ${ }^{11}$

11 Insbesondere Jaworska (2007), 529-568, hat auf die normative Bedeutung von „Caring“ aufmerksam gemacht. Sie bindet die Zuschreibung eines moralischen Status an diese Einstellung, die auch Klein- 
So verweisen entwicklungspsychologische Studien bei ca. Zweijährigen verschiedene Arten des „Caring“ nach: so etwa, wenn ein Kleinkind versucht, seine Mutter zu trösten, oder immer wieder versucht, eine bestimmte Aufgabe (wie zum Beispiel die Schuhe zu binden) ausführt. Beides sind Versuche, die häufig wenig erfolgreich sind. Sie werden jedoch von der Freude begleitet, sie selbst auszuführen. Das Kind zeigt Begeisterung, wenn ihm doch einer seiner Versuche gelingt oder es auf positive Reaktionen stößt. Es zeigt Frustration, Ärger und Wut, wenn man es davon abhält und die Aufgabe selbst erledigen will. Etwas ältere Kinder zeigen diese Art des sich Kümmerns in vielfältigen Zusammenhängen - so etwa, wenn eine Vierjährige immer wieder ins Schwimmbad möchte und entsprechende emotionale Reaktionen zeigt.

Was die Art des sich Kümmerns von bloßen Wünschen unterscheidet, ist, dass es sich bereits um eine komplexere Menge von emotionalen Einstellungen handelt, die durch ihren Fokus auf dasselbe Objekt oder denselben Sachverhalt verbunden sind, immer wieder hervorgerufen werden und dem Kind, das sie hat, aus diesem Grunde bereits verstärkt „eigen“ sind. Sie sind keine blinden Impulse, sondern setzen bereits einen (wenn auch nicht höherstufigen) Sinn von sich selbst voraus. ${ }^{12}$ Dies tun sie, indem sie psychische Verbindungen schaffen, deren Funktion darin besteht, dieses Verständnis von sich als einem Wesen, das sich über Zeit orientiert und dem bestimmte Dinge, Erfahrungen und Handlungen wichtig werden, zu konstituieren. Genauso setzen sie im Gegensatz zu blinden Leidenschaften voraus, dass das Kind ein grobes Verständnis einer relevanten Situation hat, auf die es reagiert. Emotionen können genau diese Rolle spielen. Indem verschiedene emotionale Einstellungen auf dasselbe Objekt oder denselben Sachverhalt in Abhängigkeit von einem relevanten Kontext gerichtet sind, wird nicht nur das Selbstverständnis eines Kindes zunehmend entwickelt, sondern die Einstellungen werden auch verständlich als Ausdruck dessen, was dem Kind etwas bedeutet. Ein kleines Kind kann sich jedoch um etwas kümmern, ohne sich dessen selbst bewusst zu sein und ohne einen Begriff des Guten zu besitzen. Weder die Rechtfertigungsbedingungen seines Kümmerns sind ihm zugänglich, noch fällt es Urteile darüber. Die Einstellung des sich Kümmerns erfüllt somit nur die zweite von Schefflers Bedingungen für Wertschätzen.

Wie gelangen Kinder jedoch dazu, eigene Wertungen auszubilden? Warum sollten sie sie ausbilden? Und warum sollten ihre Wertungen - sofern sie sie erwerben - auf der Proto-Form des sich Kümmerns aufbauen? Um mit der letzten Frage zu beginnen, und in Anlehnung an die Arbeit von Agnieszka Jaworska zur Bedeutung dieser Vorform des Wertschätzens, stellt sich Kümmern den basalen Baustein für eine evaluative Perspektive eines Kindes dar. Allerdings kann das Kind fehlgehen in der Einschätzung dessen, worum es sich kümmert und wie es sich darum kümmert. Es kann sich um Dinge kümmern, deren Wert eine solche Einstellung nicht verdient. Und es kann noch nicht bewusst sein Kümmern als normative Quelle des eigenen Tuns betrachten und sich entsprechend selbst kontrollieren. Es ist jedoch der erste Baustein, weil das Kind auf diese Weise seine Empfänglichkeit auf ein Objekt stabil zu lenken vermag und sich selbst als gegenüber diesem Objekt verwundbar erfährt. Um jedoch einmal auf das wertend zu reagieren, was wertvoll ist, und um sich entsprechend im Lichte dessen zu führen (auch gegen anderweitige Neigungen), sollte ein Kind lernen, wertzuschätzen. Nur

kinder und Alzheimer-PatientInnen aufweisen. Im Folgenden geht es mir lediglich darum, Caring als Protoform des Wertschätzens zu spezifizieren.

12 In diesem Sinne führt Jaworska (2007), 552, aus: ,,any attitude, reflexive or not, has the right kind of authority to speak for the agent, so long as it is part of its function to support the psychological continuities and connections that constitute the agent's identity and cohesion over time." 
dann erfüllt es die für Autonomie zentrale Authentizitätsbedingung. Nur dann versteht es sein Wertschätzen als gerechtfertigt, kann sich bewusst daran orientieren und ein bewusstes Verständnis seiner selbst entwickeln.

Doch dies geschieht nicht von selbst. Ein Kind mag zwar durch Erfahrung mehr darüber lernen, was wertvoll ist, und an den Reaktionen anderer ablesen, wann seine Reaktionen als angemessen gelten. Doch wäre es allein auf sich gestellt, würde es häufig in die Irre gehen, und mitunter einfach auch nicht die Gelegenheit haben, auf das wertend zu reagieren und sich entsprechend zu verstehen, was auch wertvoll ist. Statt sich im Lichte seiner Wertungen führen zu können, würde es zu viele Experimente des Wertens ausführen müssen oder aber gar nicht in die Lage kommen, solche Experimente zu machen. Genauso wenig würde seine Autonomie befördert, wenn Eltern einfach vorgeben, was wertvoll ist, und das Kind veranlassen, darauf zu reagieren. Denn worauf man emotional reagieren kann, ist nicht in der Verfügung anderer. Ich werde daher im Folgenden einen Vorschlag zu verteidigen suchen, wie Kinder lernen können, vor dem Hintergrund ihrer Einstellung des sich Kümmerns selbst etwas wertzuschätzen. Dies ist jedoch nur möglich, da sie bereits die Fähigkeit des sich Kümmerns mitbringen. Eltern können sich daher bereits in dieser frühkindlichen Phase bemühen, diese Einstellung zu befördern.

\section{Persönliche Projekte und eigene Wertungen}

Was Kinder des Weiteren brauchen sind Gelegenheiten, um (i) eigene Erfahrungen mit wertvollen Dingen zu machen; um (ii) diese Erfahrungen systematisch zu machen, denn um wertschätzen zu können, ist Übung erforderlich; und um (iii) sich zunehmend als Akteure mit einer evaluativen Perspektive verstehen zu lernen, um ihr Handeln entsprechend auszurichten und um zu ihren erworbenen Wertungen zu stehen beziehungsweise sie im Lichte anderer Gründe zu revidieren.

Meine These, die ich im Folgenden zunächst näher erläutern werde, ist, dass persönliche Projekte $^{13}$ genau diese Gelegenheiten bieten. Sofern Eltern also eine Pflicht haben, die Autonomie ihrer Kinder zu befördern, haben sie eine Pflicht, ihre Kinder persönliche Projekte verfolgen zu lassen. Die Gelegenheit zu geben, persönliche Projekte zu finden und zu verfolgen, ist eine Einflussnahme, die nicht autonomie-unterminierend ist. Dies ist deshalb der Fall, weil Kinder ihre Fähigkeit zu eigenen Wertungen, die bereits in der Fähigkeit zum sich Kümmern angelegt ist, durch Projekte aktualisieren können. Sie können lernen, dass ihnen etwas wirklich wichtig ist, ohne dass das, was ihnen wichtig wird, fremdbestimmt ist. Das Verfolgen von Projekten hilft ihnen hierbei, die Rechtfertigungsbedingungen ihres Kümmerns zu erwerben, sich selbst über das, was ihnen wichtig ist, zunehmend verstehen zu lernen und sich bewusst daran $\mathrm{zu}$ orientieren.

Doch was sind persönliche Projekte? Beginnen wir mit einigen Beispielen. Berufe, Hobbies, andere Freizeitbeschäftigungen, Engagements und persönliche Beziehungen sind persönliche Projekte, die trotz ihrer oberflächlichen Verschiedenheit durch die folgenden drei Elemente charakterisierbar sind. ${ }^{14}$ Projekte sind komplexe Ziele, die

13 Persönliche Projekte sind solche, die von einzelnen Personen verfolgt werden (etwa im Gegensatz zu Gruppen- oder Firmenprojekten). Wenn ich im Folgenden der Einfachheit halber von „Projekten“ spreche, meine ich immer ,persönliche Projekte“.

14 In beziehe mich hier auf Betzler (im Erscheinen). 
(i) durch Normen bestimmt sind;

(ii) Quelle zweier Arten von projekt-abhängigen Gründen sind;

(iii) durch eine identitätskonstitutive Bindung gekennzeichnet sind.

So wird ein Projekt, wie zum Beispiel der Schutz des Regenwaldes, von Normen regiert, die mehr oder weniger bestimmt vorschreiben, worin ein Projekt besteht. Diese Normen sind konstitutiv für ein Projekt. Wer sich für den Schutz des Regenwaldes engagiert, muss wissen, wie dieser Schutz bewirkt werden kann, und muss eine relevante Menge dafür einschlägiger Tätigkeiten ausführen. Wenn einmal ein Projekt verfolgt wird, dann gibt es Anlass zu spezifischen Handlungsgründen sowie zu Gründen für bestimmte emotionale Einstellungen. Wenn man zum Beispiel Fußballspielen als sein Hobby begreift, dann hat man Gründe für die wiederholte Ausführung indirekt verbundener, unterschiedlicher Handlungstypen, die gemeinsam dieses Projekt ausdrücken. So wird ein Kind immer wieder ins wöchentliche Training gehen, aber auch Fußballzeitschriften studieren, sich für Spiele guter Vereine interessieren und diese ansehen, und bei Turnieren mitspielen. Diese verschiedenen Handlungstypen beziehen sich nicht unmittelbar aufeinander, sondern sind durch die Norm verbunden, die das Projekt des Fußballspielens mehr oder weniger bestimmt definiert.

Darüber hinaus hat man, sobald man dieses Hobby hat, Gründe für verschiedene Emotionen, die das Fußballspiel als Fokus teilen. Diese Emotionen richten sich zum Teil auf wertvolle Eigenschaften dieses Hobbies, aber ebenso darauf, wie es sich in Abhängigkeit von den Umständen für die Person, die dieses Hobby verfolgt, entwickelt. So wird zum Beispiel auch ein kleiner Junge traurig, wenn er krank ist und nicht zum Training gehen kann. Er ist frustriert, wenn seine Mannschaft nicht gewinnt, freut sich, wenn er ein Tor oder einen guten Pass spielt, und ist gespannt auf das nächste Turnier. Diese über den Fokus des Hobbies und der Bedeutung dieses Hobbies für ein bestimmtes Kind verbundenen Emotionen lassen das Hobby dem Kind wichtig sein.

Je mehr ein Kind ein solches Projekt verfolgt und auf die genannten projektabhängigen Gründe reagiert, desto mehr lernt es, sich darüber zu verstehen und sich zum Beispiel selbst als Fußballspieler zu identifizieren. Es beginnt auf Grund der Tatsache, dass ihm dieses Projekt immer wichtiger wird, dieses für wertvoll zu halten. Zunehmend wird auch sein Verständnis wachsen, warum es dieses Projekt für wertvoll hält und Gründe dafür benennen können. Dieser Prozess wird zum Teil durch reflexive Emotionen ausgedrückt, wie Stolz und Selbstvertrauen.

Neben den substanziellen Eigenschaften dieser beiden genannten projektabhängigen Gründe, die bestimmte Handlungen und Emotionen nahe legen, sind sie auch durch formale Eigenschaften charakterisiert. Es handelt sich um Gründe, das eigene Tun diachron zu strukturieren und eine stabile evaluative Orientierung zu erwerben. Diese Gründe sind modal stringent: Sie lassen das Projekt nicht-instrumentell wertschätzen und die Person zunehmend daran gebunden sein. Dies zeigt sich zum Beispiel daran, dass auch ein Kind sein Hobby nicht gleich aufgibt, selbst wenn es nicht gewinnt, sich über seinen Trainer ärgert oder andere negative Erfahrungen macht.

Wenn diese Analyse, die im Rahmen dieses Beitrags nur knapp vorgestellt werden kann, überzeugt, gilt es weiter zu fragen, worin der Gehalt der Elternpflicht besteht, Kindern die Gelegenheit zum Verfolgen eines Projekts zu geben. Eltern kennen in der Regel die Talente, Vorlieben und das, worum sich das Kind bisher im oben genannten Sinn kümmert, am besten. Eltern können auf Grund ihres Wissens eine Auswahl an Projekten zur Verfügung stellen und dem Kind Gelegenheit geben, diese auszuprobieren. Darüber hinaus können sie selbst vorleben, wie sie Projekte wertschätzen. Sie können ihr Kind im Verfolgen eines Projekts ermuntern, Interesse für das Projekt ihres Kindes zeigen und ihre eigene Wertschätzung aus- 
drücken. Sie können sich mit dem Kind über die evaluativen Eigenschaften verschiedener Projekte austauschen und ihr Wissen sowohl über bestimmte Werte als auch über bestimmte Erfahrungen mit Projekten weitergeben. Dies sind Voraussetzungen dafür, dass ein Kind lernt, Projekte wertzuschätzen.

Nicht alle Arten von Projekten sind gleichermaßen für Kinder geeignet. So sind weder Berufe noch politische Aktivitäten Projekte, die für Kinder in Frage kommen. Gleichwohl gibt es eine ganze Reihe von Hobbies, Freizeitbeschäftigungen, andere handwerkliche, künstlerische oder sportliche Tätigkeiten sowie Freundschaften ${ }^{15}$, die Kindern in besonderer Weise helfen, die damit verbundenen Werte selbst schätzen zu lernen und als wichtig zu erachten. Es genügt für meine These, dass es eine Reihe von Projekten gibt, die für Kinder einschlägig sind, um zeigen zu können, dass Projekte in besonderem Maße helfen, die Autonomie eines Kindes durch die Erfüllung der dafür erforderlichen Authentizitätsbedingung zu befördern.

Nun mag an dieser Stelle eingewendet werden, dass Kinder auch ohne Projekte Dinge wertschätzen können. Inwiefern sind Projekte also notwendig, um wertschätzen zu lernen? Die Einstellung des Wertschätzens stellt sich in seltenen Fällen plötzlich ein. Projekte stellen normabhängige Handlungs- und Erfahrungsmuster dar, die einem Kind in besonderer Weise erlauben, graduell die Einstellung des Wertschätzens zu erwerben. Persönliche Projekte sind somit nicht logisch notwendig für Autonomie, aber sie sind in der Regel konstitutiv für Autonomie. Persönliche Projekte befördern diese in mehrfacher Weise:

Da es sich erstens um kulturell und sozial vorbestimmte Handlungsmuster handelt, erlauben sie in der Regel die Erfahrung mit zahlreichen wertvollen Eigenschaften verschiedener Tätigkeiten, wie etwa ästhetische, soziale, naturbezogene, handwerklich-technische, intellektuelle, kinästhetische Erfahrungen. Diese Erfahrungen können zum einen als unmittelbar positiv erlebt werden. Zum andern können Frustrationen in solchen Erfahrungen als Möglichkeit betrachtet werden, Stärke zu entwickeln und Widerstände zu überwinden. Zudem kann ein Kind die Erfahrung machen, sich durch das kontinuierliche Engagement in seinen Fähigkeiten zu verbessern, diese Verbesserung als Ergebnis der eigenen Anstrengung zu erleben und die mit einem bestimmten Projekt verbundenen wertvollen Eigenschaften zunehmend schätzen zu lernen. Dies führt zu einem Erwerb von Wissen darüber, was der Wert von verschiedenen Projekten ist.

Als Unternehmungen, die zu indirekt verbundenen wiederholten Handlungstypen Anlass geben, vermitteln sie zweitens die Möglichkeit, sich diachron an einer Sache zu orientieren und dieser gegenüber anderen Tätigkeiten eine gewisse Priorität zu geben. Kinder lernen auf diese Weise, sich selbst als diachrone Akteure wahrzunehmen, die Dinge für ihre eigene Zukunft tun können.

Dadurch geben Projekte drittens Gelegenheit, sich wertend an sie zu binden und sich graduell selbst über sie zu verstehen. Dies ist vor allem dann der Fall, wenn die Erfahrung der wertvollen Eigenschaften, die sie vermitteln, geschätzt und durch die wiederholte Ausführung vertieft werden kann. Auf diese Weise erfährt das Kind, dass sein Engagement Sinn hat und sein Leben orientiert.

Das Verfolgen persönlicher Projekte hilft neben der Möglichkeit des nicht-instrumentellen Wertschätzens viertens auch bei der Ausbildung bestimmter Tugenden, Fertigkeiten und sozialer Kompetenzen. So lernen Kinder Willensstärke und Ausdauer im Verfolgen eines Projekts. Sie können die Fähigkeit erwerben, mit Frustrationen und Niederlagen umzugehen und sich loyal zu binden. Projekte vermitteln verschiedene Fertigkeiten, je nach Art des

15 Mullin (2007), 542 ff., argumentiert, dass Liebe zu anderen Personen eine Quelle „lokaler“ Autonomie bei Kindern ist. 
Projekts. So lernt ein Kind, das Fußballspielen als Hobby verfolgt, nicht nur die wertvollen Eigenschaften dieses Sports schätzen (sich immer geübter in bestimmten Weisen zu bewegen und mit dem Ball umzugehen). Es erwirbt auch soziale Kompetenzen, wie Fairness, sich als Mitglied eines Teams zu verstehen, den Erfolg anderer zu würdigen und Beziehungen unter Gleichaltrigen zu pflegen.

Ich hoffe hinreichend gezeigt zu haben, dass persönliche Projekte tatsächlich besonders geeignete Quellen des Wertschätzens sind. Damit sind sie zugleich wichtige Bausteine zur Realisierung der für Autonomie relevanten Authentizitätsbedingung. Indem Kinder wiederholt und zusammenhängend Tätigkeiten ausführen und entsprechende Erfahrungen machen, erhalten sie die Gelegenheit, sich wertend zu binden. Ob ihnen dies gelingt, liegt allein an ihrer eigenen emotionalen Empfänglichkeit. Stellt sich diese ein, dann können sie sich kohärent über die Zeit orientieren, ihr Verständnis ihrer selbst über das stärken, was ihnen wichtig geworden ist, und eine evaluative Perspektive erwerben.

Indem Eltern ihren Kindern die Gelegenheit geben, Projekte zu verfolgen und sie darin ermutigen und sich dafür interessieren, helfen sie ihnen, eigene Wertschätzungen von tatsächlich wertvollen Eigenschaften eines Projekts zu entwickeln. Auf diese Weise können sich Kinder Werte selbst aneignen, und zwar auch dann, wenn sie noch keine vollständig ausgebildeten rationalen Fähigkeiten besitzen. Das Rätsel der Erziehung zur Autonomie kann insofern gelöst werden, als es die Kinder selbst sind, die zeigen, ob sie bestimmte Werte zu schätzen in der Lage sind - und zwar auch dann, wenn bestimmte Projekte von den Eltern vorgegeben werden. Die Eltern bestimmen sie solange nicht in einem autonomieunterminierenden Sinne fremd, solange es dem Kind selbst obliegt, ob es ein Projekt wertschätzen kann. Die Pflicht der Eltern besteht folglich nicht darin, ein Kind zu einem bestimmten Projekt zu zwingen oder andere Projekte, gegen die keine starken Gründe sprechen, zu verbieten. Ihre Pflicht besteht darin, Gelegenheiten dafür zu schaffen, dass ihre Kinder verschiedene Projekte ausprobieren können, und sie darin zu bestärken. Der Gehalt ihrer Pflicht besteht folglich, zumindest zum Teil, darin, die Möglichkeit zum Wertschätzen und damit zum Erwerb eigener evaluativer Einstellungen über die Zeit hinweg zu geben.

\section{Einwände und Erwiderungen}

Bisher habe ich meine These, dass die Gelegenheit zum Verfolgen persönlicher Projekte ein für die Autonomie des Kindes konstitutiver Bestandteil ist, lediglich etabliert und versucht plausibel zu machen. Im Folgenden möchte ich sie weiter verteidigen, indem ich sie gegen verschiedene Einwände zu stärken und zu schärfen versuche.

Erster Einwand: Überforderung. Meine These könnte den Eindruck erwecken, dass Kinder heillos überfordert werden. Insbesondere kleinere Kinder verfolgen selten persönliche Projekte. Sie besitzen weder die Ausdauer noch die Fertigkeiten und Fähigkeiten, um ein Projekt sinnvoll verfolgen zu können. Meistens beginnen sie mit einer Aktivität, um sie häufig wieder $\mathrm{zu}$ beenden und anderen Tätigkeiten nachzugehen.

Es ist tatsächlich nicht der Fall, dass (vor allem kleinere) Kinder in vollkommener Weise Projekte verfolgen. Sie reagieren bei weitem nicht auf alle projektabhängigen Gründe und bilden auch kein explizites Selbstverständnis über ihr Projekt aus. Sie verfolgen vielmehr Proto-Projekte. Das heißt, sie probieren sich noch viel mehr in unterschiedlichen Aktivitäten aus, wechseln von einem Proto-Projekt zum nächsten, oder versuchen erst herauszufinden, was genau ihr Projekt ist. Dies ist etwa der Fall, wenn ein Kind sich im Spielen verschiedener 
Instrumente versucht, um schließlich diese musikalischen Betätigungen ganz aufzugeben, weil die Freundin nun auch etwas anderes macht. Das vermeintliche musikalische Proto-Projekt mag sich so als ein Proto-Projekt in Freundschaft entpuppen.

Doch das Ausprobieren in verschiedenen Proto-Projekten hilft Kindern genau auf dem Weg zur Entwicklung stabilerer, eigener Wertungen. Sie können dadurch beharrlicher und mutiger werden, mehr Rückschläge aushalten, mehr Fertigkeiten erwerben und sich selbst besser kennen lernen, bis sie sich schließlich graduell immer mehr einem Projekt widmen können. Dass ihnen dies gelingt, hängt zwar auch noch von anderen Faktoren ab - wie etwa ihrem Charakter, der Persönlichkeit ihrer Trainer und Lehrer, die soziale Anerkennung eines Projekts durch Gleichaltrige oder die Rollenmodelle, die ihnen zu Verfügung stehen. Aber auch unabhängig von diesen anderen Faktoren besteht ein konstitutives Verhältnis von persönlichen Projekten und der Authentizitätsbedingung der Autonomie. Damit ein Kind zunehmend autonom wird und zunehmend in der Lage ist, selbst etwas wertzuschätzen und eine identitätskonstitutive Basis dafür erwirbt, sich selbst im Lichte seiner Wertungen zu führen, bedarf es vieler Möglichkeiten, Projekte auszuprobieren. Proto-Projekte - wie etwa ein einwöchiger Schwimmkurs oder ein Feriencamp mit behinderten Kindern - geben ihnen die Gelegenheit, dies zu tun. Typischerweise beginnen erst Jugendliche Projekte in einem volleren Sinne wertzuschätzen. Sofern einem Kind die Gelegenheit gegeben wird, Proto-Projekte auszuprobieren, wird es nicht überfordert, sondern an das Verfolgen von Projekten herangeführt.

Zweiter Einwand: Falsche Weisen, Projekte zu verfolgen. Selbst wenn zugestanden wird, dass Kinder sich erst graduell dazu entwickeln, Projekte wertschätzen zu können und somit eigene Einstellungen zu erwerben, so ist der Verdacht noch nicht vollständig ausgeräumt, dass Projekte die Autonomie eines Kindes nicht befördern, sondern unterminieren. Dies liegt zum einen daran, dass häufig besonders ehrgeizige Eltern - nennen wir sie „Übereltern“ - ihre Kinder in einer anderen Weise überfordern. Sie lassen ihnen nicht genügend Freiheit, ihre Projekte so zu verfolgen, wie dies den Neigungen des jeweiligen Kindes entspricht. Stattdessen üben sie ihren Einfluss dahingehend aus, dass sie ihre Kinder dazu anhalten, zu viele Projekte zu verfolgen oder ein Projekt zu intensiv zu verfolgen.

Es käme tatsächlich einer Überforderung und unrechtmäßigen Einmischung gleich, wenn Kindern nicht die Gelegenheit gegeben wird, Proto-Projekte zu verfolgen, bis sie selbst in der Lage sind, verschiedene Projekte wertzuschätzen. Es sind dann die Kinder selbst, die durch ihre tatsächliche Wertschätzung bestimmen, wie viele Projekte und wie intensiv sie sie verfolgen. Andernfalls unterminiert dies ihre Autonomie. Es ist daher wichtig zu ergänzen, dass die Gelegenheit, Projekte zu verfolgen, nicht die einzige Bedingung für die Erziehung zur Autonomie ist. Es geht auch darum, wie das Verfolgen eines Projekts befördert wird. Kinder benötigen unabhängig von Projekten und Proto-Projekten nicht nur Zeit für das Spiel, sondern sie benötigen vor allem auch Raum, Proto-Projekte auszuprobieren. Gleichwohl könnte eine Kritikerin darauf bestehen, dass das Wertschätzen von Projekten die für Kindheit typischen Fähigkeiten zum phantasievollen, aber nicht zweckgebundenen Zeitvertreib unterminiert und es sich so um konkurrierende Autonomiekompetenzen handelt. Dies scheint mir jedoch keinesfalls zwingend. Das Ausprobieren von Proto-Projekten und Zeiten des bloßen Spielens können sich sinnvoll abwechseln und ergänzen.

Zum andern gibt es Eltern, die ihre Kinder zum Verfolgen bestimmter Projekte nötigen - entweder weil sie ihren eigenen Werten entsprechen oder weil es sich um sozial besonders anerkannte Projekte handelt. Vor allem kleinere Kinder wählen ihre Projekte häufig nicht selbst aus. Vielmehr ist es in vielen Fällen sogar so, dass sie sehr dazu angehalten werden. 
Was ihre Autonomie unterminiert, ist jedoch nicht die Tatsache, dass Eltern eine Vorauswahl von Projekten treffen, zu denen sie ihren Kindern Gelegenheit geben. Was ihre Autonomie untergraben kann, ist erstens, dass ihre eigenen Interessen und Neigungen zu bestimmten Projekten unberücksichtigt bleiben (sofern keine starken Gründe dagegen sprechen ${ }^{16}$ ). Zum Zweiten wird ihre Autonomie missachtet, wenn sie weiterhin gezwungen werden, ein Projekt $\mathrm{zu}$ verfolgen, selbst wenn sie nicht in der Lage zu sein scheinen, es wertzuschätzen, oder wenn ihnen nicht genügend Zeit für Spiel und andere Tätigkeiten gegeben wird. Zum Dritten handelt es sich um nicht gerechtfertigte Einflussnahme, wenn Eltern Projekte vor allem mit dem Motiv auswählen, ihr eigenes Ansehen zu erhöhen. Es erschwert Kindern das nichtinstrumentelle Wertschätzen maßgeblich, wenn ihnen zu verstehen gegeben wird, dass sie ein Projekt auf Grund eines anderen, projektexternen Ziels - nämlich das Ansehen der Eltern und damit einhergehend deren Tadel - verfolgen müssen. Doch auch hier bleibt festzuhalten, dass - selbst wenn Eltern ihre Projekte aufzwingen - Kinder diese selbst wertschätzen können und die Authentizitätsbedingung auch bei vorgegebenen Projekten erfüllt sein mag. Ich halte es jedoch für weniger wahrscheinlich, dass Kinder bei starkem Zwang zu nicht-instrumentellem Werten in der Lage sind.

Dritter Einwand: Projekte sind kein notwendiges Mittel zur Beförderung von Autonomie. Eine Kritikerin könnte zudem darauf hinweisen, dass Projekte entweder kein allgemein verfügbares Mittel oder nicht das richtige Mittel sind, um Autonomie zu befördern. So könnte eingewendet werden, dass es sich hierbei um typische Vorstellungen einer partikularen, aber nicht weithin geteilten Auffassung von einem eigenen Leben handelt. Dabei werde zu viel Gewicht auf Leistung, Zielstrebigkeit und Eigenständigkeit gelegt, wie es für Wohlstandsgesellschaften typisch ist. Dies scheint mir jedoch ein falsches Verständnis von Projekten zu sein. So besteht das konstitutive Ziel von Projekten gerade nicht darin, projektunabhängigen Erfolg oder fremdbestimmte Leistungskraft zu befördern, sondern ein Projekt um seiner selbst willen, das heißt nicht-instrumentell, wertzuschätzen. Abgesehen davon, dass dies nicht durch äußeren Druck erreicht werden kann, können auch Unternehmungen, die gemeinhin nicht mit Leistung und Erfolg assoziiert werden, wie etwa Meditieren, einen Garten pflegen oder ein Baumhaus bauen (um nur drei Beispiele herauszugreifen), als Projekte dienen. Sie geben genauso Anlass $\mathrm{zu}$ indirekt verbundenen Handlungstypen und verbundenen Emotionen und können in diesem Sinne wertgeschätzt werden, ohne dass Erfolg und Leistung je eine motivationale oder projektunabhängige Rolle spielen müssten.

Eine eigene Einstellung im Wertschätzen eines Projekts zu entwickeln, heißt auch nicht, dass dies eine isolierte Betätigung sein muss. Es können ganz im Gegenteil auch gemeinsame Projekte - wie etwas das Musizieren in einem Orchester - geschätzt werden. Mit „eigen“ ist lediglich gemeint, dass das Kind selbst sich als gegenüber diesem Projekt berührbar erweisen muss.

Ebenso und nicht unabhängig davon könnte eingewendet werden, dass die Forderung, Eltern sollen das Verfolgen von Projekten befördern, inhärent unfair ist. Schließlich könnten dann nur Kinder wohlhabender Eltern - solche nämlich, die sich den extracurricularen Ballett- und Klavierunterricht leisten können - autonom werden. Eine plausible Moraltheorie könne aber keine Pflichten formulieren, die eine große Gruppe derer, die ein Recht auf die Erfüllung dieser Pflichten haben, kontingenterweise ausschließt. Eine solche Moraltheorie wäre selbstaufhebend, sofern sie den Ausschluss der Adressaten moralischer Forderungen fordert. Es ist jedoch ein Fehler zu glauben, dass Projekte notwendigerweise kostspielige

16 Dies wäre zum Beispiel bei unmoralischen oder riskanten Projekten der Fall. 
Kurse erfordern oder konsumorientierte Unternehmungen sein müssen. Es geht nicht darum, Kindern zu sozial angesehenen (und daher häufig teuren) Projekten zu bewegen. Es geht darum, dass Kinder in die Lage versetzt werden, Projekte wertzuschätzen. Dazu kann auch gehören, mit Nachbarskindern täglich Fußball zu spielen.

Darüber hinaus könnte man der Ansicht sein, Projekte wären ein ungeeignetes Mittel zur Erlangung von Autonomie. Dies liege unter anderem daran, dass die Forderung, Projekte zu verfolgen, selbstrechtfertigend ist. Wenn ein Kind ein bestimmtes Projekt wertschätzt, hat es einen Grund erworben, dieses Projekt weiterzuverfolgen, und wird auf diese Weise zunehmend autonom. Das Projekt würde dann einen Grund geben, und zwar unabhängig davon, ob es sich um ein tatsächlich wertvolles Projekt handelt. So können schließlich auch Projekte, wie ,andere Kinder hänseln“, „Tiere quälen“, „Spielsachen stehlen“ oder „ohne Führerschein mit dem Auto der Eltern fahren“, zu indirekt verbundenen Handlungstypen und verbundenen Emotionen veranlassen. Ebenso - und dies scheint für Pubertierende typisch - können merkwürdige und vor allem Eltern als ,fremd“ anmutende Projekte verfolgt werden. Eine Dreizehnjährige mag sich zum Beispiel nur noch für „Gothic Culture“ interessieren und dies als Projekt verfolgen.

Ich gestehe hierbei zum einen zu, dass es tatsächlich unmoralische, unfaire und unkluge Projekte gibt. Gegen solche Projekte sprechen starke Gründe - nämlich moralische Erwägungen, Fairness- oder Klugheitserwägungen. Ich halte dennoch daran fest, dass allein das Wertschätzen eines Projekts selbst einen Grund generiert, ein solches Projekt weiterzuverfolgen - nämlich in dem Maße, in dem es qua Projekt eine Gelegenheit zu wertvollen Erfahrungen, verbundenen Emotionen und Tätigkeiten über die Zeit gibt, die konstitutiv für die Identität eines Kindes und Ausdruck einer dem Kind eigenen Einstellung sind. Dass dies einen Grund generiert, heißt nicht, dass es sich um einen Grund in Anbetracht aller Umstände handelt. Es handelt sich um einen Grund in den genannten Hinsichten, der von anderen Gründen, wie den oben genannten, überwogen werden kann. Zugleich halte ich zum andern daran fest, dass auch die merkwürdigsten Projekte Anlass geben, eigene Wertungen auszubilden. Gerade die für das Pubertätsalter typischen, zum Teil für Eltern schwer aushaltbaren Projekte ermöglichen es, dass Jugendlichen etwas selbst wirklich wichtig werden kann, und zwar gerade in Abgrenzung zu ihren Eltern.

Vierter Einwand: Projekte sind nicht hinreichend für Autonomie. Autonomietheoretiker verschiedener Couleur mögen darauf hinweisen, dass das Verfolgen von Projekten nicht hinreichend für Autonomie ist. Diesem Hinweis stimme ich zu. Es gibt andere relevante Faktoren, die für Autonomie notwendig sind, wie zum Beispiel Freiheit von Manipulation und innerem Zwang oder kritische Reflexionsfähigkeit. Meine These ist nur, dass Projekte eine relevante Gelegenheit bieten, verschiedene wertvolle Eigenschaften schätzen zu lernen, und aus diesem Grunde eine wichtige Basis bilden, um zum einen die Fähigkeiten zu erwerben, die für Autonomie notwendig sind, aber auch um diese Fähigkeiten in einem für Autonomie relevanten Sinne selbst zu erwerben.

Sofern Projekte für die Autonomie eines Kindes notwendig sind, so ist Autonomie kein vom Verfolgen eines Projekts unabhängiges Resultat, das angestrebt werden kann. Projekte - sofern sie helfen, die Authentizitätsbedingung von Autonomie zu erfüllen - sind vielmehr konstitutiv für Autonomie. Sofern es gelingt, ein Projekt wertzuschätzen und sich entsprechend zu orientieren, können sie ausdrücken, was einem Kind wirklich wichtig ist und wie es sich versteht. Insofern tragen sie dazu bei, dass ein Kind die für Autonomie notwendige Authentizitätsbedingung zunehmend erfüllt. 


\section{Konklusion}

Ich habe im Rahmen dieses Beitrags nicht alle möglichen Einwände ausräumen können. Es gibt auch noch weiteren Bedarf, den Begriff eines Projekts zu schärfen und gegenüber anderen Zielen zu präzisieren. Ich hoffe jedoch, meine These, dass das Wertschätzen von Projekten ein für Autonomie konstitutives Mittel ist, hinreichend plausibilisiert zu haben. Ich habe hierbei versucht zu zeigen, dass das Rätsel der Erziehung zur Autonomie gelöst werden kann, indem Eltern ihren Kindern die Gelegenheit zum Verfolgen und etwaigen Wertschätzen von Projekten geben sollen. Da die Wertschätzung eines Projekts von den Einstellungen des Kindes abhängt, ist es das Kind selbst, das lernt, wertzuschätzen. Auf diese Weise erwirbt es eine eigene evaluative Basis, um sich selbst entsprechend zu führen. Die autonomieunterminierende Einflussnahme der Eltern kann dadurch vermieden, die Erziehung zur Autonomie eines Kindes aber dennoch befördert werden. Das Rätsel der Erziehung zur Autonomie ist dadurch gelöst. ${ }^{*}$

Prof. Dr. Monika Betzler, Universität Bern, Institut für Philosophie, Länggassstrasse 49a, 3000 Bern 9, Schweiz

\section{Literatur}

Betzler, M. (2009 / erschienen 2011), Macht uns eine Veränderung unserer selbst autonom? Überlegungen zur Rechtfertigung von Neuro-Enhancement der Emotionen, in: Philosophia Naturalis, 46, $167-212$.

Dies. (im Erscheinen), Persönliche Projekte als diachrone Orientierungsprinzipien, in: D. Sturma (Hg.), Julian Nida-Rümelin über Vernunft und Freiheit, Berlin.

Brennan, S. (2002), Children's Choices or Children's Interests: Which Do Their Rights Protect, in: C. Macleod u. D. Archard (Hg.), The Moral and Political Status of Children: New Essays, Oxford.

Brighthouse, H. (1998), Civic Education and Liberal Legitimacy, in: Ethics, 108, 719-745.

Christman, J. (2009), The Politics of Persons: Individual Autonomy and Socio-Historical Selves, Cambridge.

Cuypers, S. E. u. I. Haji (2007), Authentic Education and Moral Responsibility, in: Journal of Applied Philosophy, 24, 78-94.

Dworkin, R. (2010), Justice for Hedgehogs, Cambridge/Mass.

Feinberg, J. (1980), The Child's Right to an Open Future, in: W. Aiken u. H. LaFollette (Hg.), Whose Child? Children's Rights, Parental Authority and State Power, Totowa, 124-153.

Jaworska, A. (2007), Caring and Internality, in: Philosophy and Phenomenological Research, LXXIV, $529-568$.

Lomasky, L. (1984), Personal Projects as the Foundation for Basic Rights, in: Social Philosophy \& Policy, 1, 35-55.

Mullin, A. (2007), Children, Autonomy, and Care, in: Journal of Social Philosophy, 38, 536-553.

* Für hilfreiche Diskussionen und Kommentare zu einer früheren Version dieses Beitrags danke ich Holger Baumann, Barbara Bleisch, Magdalena Hoffmann und Nina Scherer. Ebenso dankbar bin ich den TeilnehmerInnen der an der Universität Zürich veranstalteten Konferenz „Encountering Children“ im Juni 2011 für hilfreiche Hinweise zu einer vorgetragenen Version dieses Beitrags. Andrea Esser danke ich für die Einladung, meine Überlegungen im Rahmen dieses Themenhefts zur Autonomie vorzustellen. 
Noggle, R. (2005), Autonomy and the Paradox of Self-Creation: Infinite Regresses, Finite Selves, and the Limits of Authenticity, in: J. S. Taylor (Hg.), Personal Autonomy: New Essays on Personal Autonomy and Its Role in Contemporary Moral Philosophy, Cambridge, 87-108.

Oshana, M. (1998), Personal Autonomy and Society, in: Journal of Social Philosophy, 29, 81-102.

Dies. (2003), How Much Should We Value Autonomy?, in: Social Philosophy \& Policy, 20, 99-126.

Richards, N. (2010), The Ethics of Parenthood, Oxford.

Scheffler, S. (2010), Valuing, in: ders., Equality and Tradition, Oxford, 15-40.

\begin{abstract}
Parents have the duty (among others) to enhance the autonomy of their child. Someone can be regarded as autonomous if she knows what she really wants, and if she is able to guide herself over time in light of that. I first show that we are confronted with a puzzle: children can be characterized, in part at least, as not being sufficiently autonomous (if at all). To enhance the autonomy of a child, it must be subject to her parents' heteronomy. But how can a child then ever find out what it really wants for herself? In a second step, I defend the claim that a child needs to acquire the attitude of valuing. I propose a definition of valuing, and show that children already are proto-valuers. I then argue that personal projects are the adequate means to acquire the attitude of valuing. In discussing four objections I bolster the view that personal projects are constitutive for the autonomy of a child. They are therefore an important part of the parents' duty to enhance the child's autonomy.
\end{abstract}

